\section{PWE-025 MICROSCOPIC COLONIC INFLAMMATION IN IMMUNE CHECK POINT INHIBITOR-INDUCED DIARRHOEA/ COLITIS}

1,2 Hajir Ibraheim* ${ }^{3}$ Lavinia Spain, ${ }^{2}$ Amit Samani, 1,2 2 Sophie Papa ${ }^{3}$ Nadia Yousaf, ${ }^{3}$ Martin Gore, ${ }^{3}$ James Larkin, ${ }^{3}$ Samra Turajili, ${ }^{1,2}$ Nick Powell. 'Guy's And St Thomas' Hospital NHS Foundation Trust, London, UK; ${ }^{2}$ Kings College London, London, UK; ${ }^{3} T$ The Royal Marsden Hospital, London, UK; ${ }^{4}$ The Francis Crick Institue, London, UK

\subsection{6/gutjnl-2018-BSGAbstracts.157}

Background Immune check point inhibitors (ICPis) have transformed the treatment landscape for several cancers, but at the cost of triggering ICPi-induced colitis which resembles some aspects of IBD. Diagnosis is often made by symptoms, or by identifying endoscopic features of colitis. Little is known about histological findings in the absence of macroscopic disease. Furthermore, first-line management strategies beyond the use of systemic corticosteroids have not been explored. Our aim was to assess the incidence of microscopic inflammation in patients with ICPi-diarrhoea, and report our experience of treating two such patients with beclomethasone diproprionate (Clipper).

Methods Electronic records of patients with advanced melanoma and ICPi- diarrhoea/colitis at the Royal Marsden Hospital (RMH) and Guy's and St Thomas' Hospital (GSTT) between 2011-2016, were retrospectively reviewed. Endoscopic, histological and clinical outcome data was recorded for patients who underwent flexible sigmoidoscopy and had colonic biopsies taken regardless of macroscopic findings.

Two symptomatic patients (one treated with anti-PD-1, and another on combination -anti-PD-1/anti-CTLA-4 therapy) with isolated microscopic disease were managed with $5 \mathrm{mg}$ Clipper, once a day, for 4 weeks. Endoscopic, histological and clinical outcomes were recorded 6 weeks after completion of therapy. Results A total of 63 flexible sigmoidoscopies were performed in 59 patients with ICPi diarrhoea/colitis. Microscopic inflammation with normal macroscopic appearances were recorded in $22 \%$ of cases. 6 patients were prescribed anti-CTLA-4, 4 anti-PD-1, and 4 combination therapy. Histological features that were distinct from conventional microscopic colitis were recorded in the majority of patients (10/14), which included acute and chronic inflammation, architectural distortion, crypt abscess formation and neutrophil infiltration. Four patients had changes consistent with conventional microscopic colitis (2 lymphocytic colitis, 2 collagenous colitis). Clipper induced clinical remission within 7 days, and histological remission by week 6 in both patients with ICPi-induced microscopic inflammation. There was no treatment associated adverse events.

Conclusion Microscopic inflammation in the absence of macroscopic features of colitis is a common finding in ICPi-induced diarrhoea, justifying the routine practice of performing colonic biopsies even when endoscopy is normal. Our favourable clinical experience of using Clipper in 2 patients with microscopic inflammation merit further investigation in appropriately controlled clinical trials.

\section{PWE-026 ENDOSCOPY IS SUPERIOR TO STOOL FREQUENCY IN PREDICTING RESPONSE TO STEROIDS IN ACUTE ULCERATIVE COLITIS}

Fiona Jagger, Rebecca Grant, Thomas Manship, Ruairi Lynch, Charlie Lees, Jack Satsangi, Ian Arnott, Nikolas Plevris*. Department of Gastroenterology, Western General Hospital, Edinburgh, UK

10.1136/gutjin-2018-BSGAbstracts.158
Introduction Acute Severe Ulcerative Colitis (ASUC) affects $15 \%$ of patients with Ulcerative Colitis. The current standard of care is intravenous (IV) steroids, with ciclosporin or antiTNF as second line medical therapy and urgent colectomy when medical therapy fails. Previous data from our centre reports $40 \%$ of those admitted with ASUC required colectomy. We aimed to determine predictors of outcome in patients admitted with acute UC.

Methods Patients admitted to NHS Lothian hospitals between $1 / 11 / 13$ and 31/10/16 requiring IV steroids for UC were identified using ICD10 discharge codes. Only the first admission for each patient during that period was included. Case record review verified the diagnosis and collected clinical variables including age, sex, disease activity (Mayo endoscopic severity score) and distribution, and treatments needed. Response to therapy was defined as discharge from hospital requiring no further anti-inflammatory treatment. Comparisons were made using Kruskal-Wallace or chi-squared analyses where appropriate.

Results 173 patients were identified (62\% male, median age 39.67 years (IQR 29.58-54.75)). 108/173 (63\%) responded to IV steroids, 29/173 (17\%) responded to second line medical therapy and 35/173 (20\%) patients required colectomy. One patient died from complications of concurrent disease.

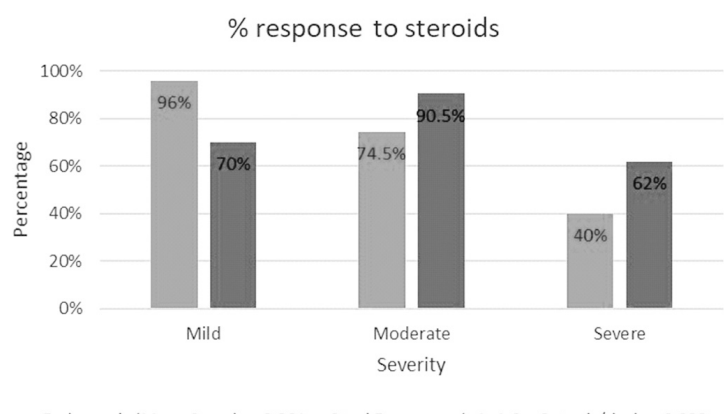

m Endoscopic (Mayo Score) $p<0.001$ Stool Frequency $(<4,4-6,>6$ stools $/$ day) $p=0.028$

\section{Abstract PWE-026 Figure 1}

Endoscopic assessment more closely reflected response to intravenous steroids than stool frequency. 96\% of those with mild endoscopic disease responded to IV steroids, compared to $40 \%$ of those with severe endoscopic disease $(p<0.001)$. $70 \%$ of those with stool frequency $<4 /$ day responded to steroid therapy compared to $62 \%$ of those with $>6$ stools/day $(p=0.028)$. Endoscopy was performed on day 2 on average (IQR 1-3) (figure 1).

Those requiring surgery had a shorter disease duration compared with those responding to IV steroids (median 0.13 years vs 1.5 years, $p=0.09$ Kruskal-Wallace). The median length of admission was 7.5 days (IQR 6-16) and surgery was performed on day 9 (IQR 8-12) on average. Age, sex, disease extent at diagnosis and smoking status were similar between groups.

Conclusions $20 \%$ of patients admitted to hospital for IV steroid treatment for active UC required urgent colectomy during admission. Response to steroids was greater in those with less severe endoscopic disease with a clearer relationship between endoscopic severity and response than stool frequency on admission. Those requiring surgery were likely to have a shorter disease duration on admission. These data emphasise the need for a timely endoscopic assessment for all patients admitted with acute colitis. 